\title{
Cetuximab plus platinum-based chemotherapy in head and neck squamous cell carcinoma: a randomized, double-blind safety study comparing cetuximab produced from two manufacturing processes using the EXTREME study regimen
}

Denis Soulières ${ }^{1}$, Jose Luis Aguilar ${ }^{2}$, Eric Chen ${ }^{3}$, Krzysztof Misiukiewicz ${ }^{4}$, Scott Ernst ${ }^{5}$, Hyun Jung Lee ${ }^{6}$, Katherine Bryant ${ }^{6}$, Shuang He ${ }^{6}$, Coleman K. Obasaju ${ }^{6}$, Shao-Chun Chang ${ }^{6}$, Steve Chin ${ }^{6}$ and Douglas Adkins ${ }^{7^{*}}$

\begin{abstract}
Background: Cetuximab, in combination with platinum chemotherapy plus 5 -fluoruracil (5-FU), is approved for the first-line treatment of recurrent/metastatic squamous cell carcinoma of the head and neck (SCCHN). Cetuximab manufactured by ImClone (US commercial cetuximab) potentially results in higher systemic exposures than cetuximab manufactured by Boehringer Ingelheim (BI-manufactured cetuximab). This prospective, randomized, double-blind study compared the safety profiles of the two cetuximab formulations.

Methods: Patients with previously untreated locoregionally recurrent and/or metastatic SCCHN were randomly assigned to receive the same dose of US commercial cetuximab (Arm A) or Bl-manufactured cetuximab (Arm B), each in combination with cisplatin or carboplatin plus 5-FU. The primary outcome was all-grade, all-cause treatment-emergent adverse events (TEAEs).

Results: The majority of patients experienced $\geq 1$ TEAE, regardless of causality (Arm A: 75/77 patients, $97.4 \%$; Arm B: 68/71 patients, $95.8 \%)$. TEAEs with the highest incidence included nausea, fatigue, and hypomagnesemia in both arms. The absolute risk difference between the two arms for patients experiencing at least one adverse event (AE) was 0.029 ( $p=0.281,95 \%$ confidence interval [CI]: - $0.024,0.082)$ for AEs regardless of causality and 0.005 ( $p=0.915$, $95 \%$ Cl: $-0.092,0.103)$ for AEs possibly related to study drug. There were no significant differences between the two arms in the incidence of acneiform rash, cardiac events, infusion reactions, or hypomagnesemia. Overall survival, progression-free survival, and overall response rates were similar in the two arms. (Continued on next page)
\end{abstract}

\footnotetext{
*Correspondence: dadkins@dom.wustl.edu

'Washington University School of Medicine, 660 S. Euclid, Box 8056, St. Louis,

MO 63110, USA

Full list of author information is available at the end of the article
}

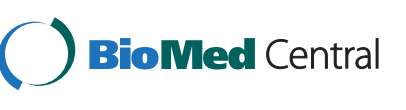

(c) 2016 Soulières et al. Open Access This article is distributed under the terms of the Creative Commons Attribution 4.0 International License (http://creativecommons.org/licenses/by/4.0/), which permits unrestricted use, distribution, and reproduction in any medium, provided you give appropriate credit to the original author(s) and the source, provide a link to the Creative Commons license, and indicate if changes were made. The Creative Commons Public Domain Dedication waiver (http://creativecommons.org/publicdomain/zero/1.0/) applies to the data made available in this article, unless otherwise stated. 
(Continued from previous page)

Conclusions: There were no clinically meaningful differences in safety between US commercial cetuximab and BImanufactured cetuximab in combination with platinum-based therapy with 5-FU in patients with locoregionally recurrent and/or metastatic SCCHN. The use of US commercial cetuximab in this combination chemotherapy regimen did not result in any unexpected safety signals. The efficacy results of this study are consistent with the efficacy results of the cetuximab arm of the EXTREME study.

Trial registration: ClinicalTrials.gov NCT01081041; date of registration: March 3, 2010).

Keywords: Carcinoma, squamous cell, Cetuximab, Head and neck cancer, Safety

\section{Background}

Head and neck cancer is the sixth most common cancer worldwide, with more than 650,000 new cases diagnosed each year [1]. Treatment options for patients with recurrent/metastatic squamous cell carcinoma of the head and neck (SCCHN) are limited. Currently, the standard of care (category 1 evidence) for recurrent/ metastatic SCCHN is the regimen used in the EXTREME study (Erbitux in First-Line Treatment of Recurrent or Metastatic Head and Neck Cancer), consisting of cetuximab in combination with cisplatin or carboplatin plus 5fluorouracil (5-FU) [2, 3]. Historically, median survival with chemotherapy is approximately 6 months and the 1 -year survival rate is approximately $20 \%$ [2]. The addition of cetuximab, an anti-epidermal growth factor receptor (EGFR) monoclonal antibody, to cytotoxic agents has shown a significant increase in response rate $[3,4]$ and a significant survival benefit [3] in recurrent/ metastatic SCCHN.

In the EXTREME study, conducted in 17 European countries, 442 patients with previously untreated recurrent/metastatic SCCHN were randomized to receive chemotherapy alone (cisplatin or carboplatin plus 5-FU) or in combination with cetuximab [3]. There were statistically significant and clinically meaningful improvements in all efficacy endpoints (overall survival $[\mathrm{OS}]$, progression-free survival [PFS], and overall response rate $[\mathrm{ORR}]$ ) in the cetuximab plus chemotherapy group compared with the chemotherapy-alone group. Overall, the safety data from the EXTREME study indicated that the addition of cetuximab to chemotherapy did not affect tolerability and that the adverse event (AE) profile for this cetuximab-chemotherapy regimen was consistent with that expected for the agents used [3]. Adverse events of interest for cetuximab include acneiform rash, cardiac events, infusion reactions, and hypomagnesemia [3, 5]. Based on the results of the EXTREME study, cetuximab was approved by the European Union (EU) for the treatment of patients with SCCHN in combination with platinum-based chemotherapy for recurrent and/or metastatic disease [6], and later by the United States (US) Food and Drug Administration (FDA) for the first-line treatment of patients with recurrent locoregional disease or metastatic SCCHN in combination with platinum-based therapy with 5-FU [5].

The cetuximab clinical supply for the EXTREME study was manufactured by the Europe-based company Boehringer Ingelheim (BI-manufactured cetuximab). Population pharmacokinetic data indicate that cetuximab manufactured by the US-based company ImClone (US commercial cetuximab) is associated with approximately $22 \%$ higher systemic drug exposure relative to BImanufactured cetuximab, due to decreased clearance [5]. Due to the potential for increased exposure and the possibility of a greater incidence and severity of adverse reactions with US commercial cetuximab compared with BImanufactured cetuximab [5], a prospective study of the two cetuximab formulations was conducted. This study compared the safety profiles of US commercial cetuximab and BI-manufactured cetuximab, each in combination with cisplatin or carboplatin plus 5-FU (the regimen used in the EXTREME study [3]), in patients with locoregionally recurrent and/or metastatic SCCHN.

\section{Methods}

\section{Study population}

The patient eligibility criteria were similar to those of the EXTREME study [3]. Patients with histologically or cytologically confirmed locoregionally recurrent and/or metastatic SCCHN not suitable for local therapy were eligible for this study. Other eligibility criteria included: age $\geq 18$ years; measurable or evaluable disease, as defined by Response Evaluation Criteria in Solid Tumors (RECIST) version 1.0; Karnofsky Performance Status (KPS) score of at least 70; and adequate hematologic, renal, and hepatic function. Patients were excluded from the study if they had: previous systemic chemotherapy, unless required as part of multimodal treatment for locally advanced head and neck cancer that was completed more than 4 months before study entry; previous treatment with monoclonal antibody therapy, other signal transduction inhibitors, or EGFR targeting therapy, except for previous cetuximab treatment given as part of a multimodal treatment for locally advanced head and neck cancer that was completed more than 4 months 
before study entry; nasopharyngeal carcinoma; or other concomitant anticancer therapies.

The study was conducted in hospital-based clinics in North America. The study protocol was approved by the ethics review board at each site (see below) and was conducted in accordance with Good Clinical Practice guidelines and the Declaration of Helsinki. All patients provided written informed consent before undergoing any study procedure. The study was registered at www.clinicaltrials.gov (NCT01081041) [7].

\section{Ethics review boards}

The following ethics review boards approved the study protocol: US Oncology, Western Institutional Review Board, Washington University Medical Center, Dallas VA Medical Center IRB, Committee for the Protection of Human Subjects, Wayne State University, Wake Forest University School of Medicine, Cleveland Clinic of Weston Florida, University of Illinois College of Medicine, Scott \& White Institutional Review Board, Decatur Memorial Hospital, Medical University of South Carolina, Stratton VA Medical Center, Veterans Affairs Medical Center, Medical College of Georgia, Translational Research in Oncology-US Inc, Mount Sinai School of Medicine Dermatology Clinical Trials, Comite Bioetico para la Investigacion Clinica SC, Centro Anticanceroso Cruz Roja Mexicana, Instituto Nacional de Cancerologia, Centro Estatal de Cancerologia, Antiguo Hospital Civil de Guadalajara, Ontario Cancer Research Ethics Board, Alberta Health Services, and Comite D'Ethique De La Recherche.

\section{Study design}

This was a multicenter, Phase 2 study with a single-arm, open-label, 30-patient safety lead-in phase followed by a two-arm, randomized, double-blind phase in patients with locoregionally recurrent and/or metastatic SCCHN who had not received prior systemic chemotherapy. The 30-patient lead-in phase was requested by the FDA to assess the safety of US commercial cetuximab before starting the randomized phase of the study.

Patients were randomly assigned to US commercial cetuximab plus chemotherapy (Arm A) or BImanufactured cetuximab plus chemotherapy (Arm B) in a 1:1 ratio (first patient enrolled: 08 June 2010). Randomization was carried out using a computergenerated random sequence and a centralized interactive voice response system (IVRS). To maintain the blinding of patients and the personnel involved in patient evaluations and data collection, an unblinded third party was designated. The investigator provided the necessary information to the unblinded designee who then called the IVRS to obtain the patient's treatment assignment.
A minimization principle was used to balance patient assignment between treatment arms, using a probability factor of 0.75 , based on the following factors: primary tumor site (oral cavity/oropharynx vs other); previous chemotherapy (yes vs no); previous cetuximab treatment (yes vs no); KPS (<80 vs $\geq 80$ ); intention to give cisplatin or carboplatin; measurable vs evaluable disease; and participating center.

\section{Treatment protocol}

Study drugs were administered intravenously in 21-day cycles, with a 1-hour observation period between cetuximab and cisplatin/carboplatin, in the following order: (i) cetuximab $400 \mathrm{mg} / \mathrm{m}^{2}$ (2-hour infusion) on Day 1 of Cycle 1 and $250 \mathrm{mg} / \mathrm{m}^{2}$ (1-hour infusion) weekly thereafter; (ii) cisplatin $100 \mathrm{mg} / \mathrm{m}^{2}$ (1-hour infusion) or carboplatin area under the concentration curve (AUC) $5 \mathrm{mg} / \mathrm{mL} / \mathrm{min}$ (1-hour infusion) on Day 1 of Cycles 1 to 6; (iii) 5 -FU $1000 \mathrm{mg} / \mathrm{m}^{2} /$ day as a continuous infusion on Days 1 through 4 of Cycles 1 to 6 . Patients enrolled in the safety lead-in phase and patients randomized to Arm A received US commercial cetuximab; patients randomized to Arm B received BImanufactured cetuximab. Patients received cisplatin or carboplatin at the discretion of the investigator. Dose modifications of cetuximab and chemotherapy were permitted according to protocol-specified criteria. Patients who completed six cycles of combination therapy continued to receive cetuximab monotherapy until disease progression, unacceptable toxicity, or any other withdrawal criterion was met. If clinically indicated, patients who discontinued chemotherapy prior to completing six cycles of combination therapy were permitted to continue with cetuximab until a withdrawal criterion was met. Patients who discontinued cetuximab were permitted to continue with chemotherapy up to six cycles.

\section{Assessments}

Adverse events were assessed according to the Common Terminology Criteria for Adverse Events (CTCAE; Version 4.0) and the Medical Dictionary for Regulatory Activities (MedDRA; Version 16.0).

Tumor response was assessed using RECIST version 1.0. Tumor measurements were performed at baseline and every two cycles by computed tomography or magnetic resonance imaging; chest $\mathrm{x}$-ray was acceptable for clearly defined lesions surrounded by aerated lung.

\section{Outcomes}

The primary objective of the study was to prospectively compare the safety profiles of US commercial cetuximab and BI-manufactured cetuximab with respect to individual all-grade, all-cause treatment-emergent adverse 
events (TEAEs) occurring at any time during the treatment period. A TEAE was defined as an event that first appeared or an event already present that worsened in severity following the first dose exposure and up to 30 days after the last dose of study treatment. Secondary objectives included overall safety, OS, PFS, and ORR.

\section{Statistical analysis}

Planned enrollment was approximately 230 patients: 30 patients for the safety lead-in phase and 200 patients for the two-arm, randomized, double-blind phase. The sample size was based on practical and clinical considerations to ensure that the safety profile could be appropriately compared between the two treatment arms and was not based on any statistical assumptions or hypotheses.

For the safety lead-in phase, the safety and efficacy analyses were conducted on the safety lead-in population, which consisted of all patients enrolled in this phase of the study. For the randomized phase, the safety and efficacy analyses were conducted on the randomized and treated (RT) population, which consisted of all patients in the two-arm, randomized, double-blind phase of the study who received at least one dose of any of the study drugs (cetuximab, cisplatin/carboplatin, and 5-FU).

The primary outcome was all-grade, all-cause TEAEs in the RT population. The risk difference for the occurrence of each $\mathrm{AE}$ was calculated and the $95 \%$ confidence interval $(\mathrm{CI})$ and $p$ value were generated using normal approximation. The false discovery rate method of multiplicity adjustment was applied to control the type I error (the false positive rate) when comparing individual AEs. Adverse events of special interest were hypomagnesemia and the composite terms acneiform rash, cardiac event, and infusion reaction. Exposure to cetuximab and chemotherapy was reported as median duration plus $25^{\text {th }}$ and $75^{\text {th }}$ percentiles and relative dose intensity (actual dose delivered as a percentage of planned dose). Overall survival was defined as the time from randomization to death; PFS was defined as the time from randomization to the first objective progression of disease or death. The Kaplan-Meier method was used to estimate median OS and PFS. Log-rank and Wilcoxon statistics were calculated to provide betweentreatment comparisons unadjusted for covariates for OS and PFS. The ORR was calculated as the percentage of patients with a confirmed (within 28 days) best response of complete response (CR) or partial response (PR). The disease control rate (DCR) was calculated as the percentage of patients with a confirmed best response of CR, PR, or stable disease. Analyses were performed using SAS version 9.2 (SAS Institute, Cary, NC, USA).
Because of manufacturing process changes, Boehringer Ingelheim stopped manufacturing the cetuximab formulation used in the EXTREME study. The supply of BImanufactured cetuximab expired during the study period; upon expiration, ongoing patients in Arm B were switched to US commercial cetuximab. For the primary safety analysis, the data analysis cut-off date was the earliest date that a patient in Arm B was switched from BI-manufactured cetuximab to US commercial cetuximab (23 January 2013). For the efficacy analyses, the data analysis cut-off date was the date that the reporting database was locked for analysis (27 September 2013). For any patient in Arm B who was switched from BI-manufactured cetuximab to US commercial cetuximab, OS and PFS were censored at the time of the switch.

\section{Results}

\section{Patient disposition}

A total of 33 patients were enrolled in the safety lead-in phase of the study and constituted the safety lead-in population. As the safety and efficacy results for the safety lead-in population were consistent with those for Arm A in the RT population (both of which received US commercial cetuximab), the results for this population are not presented here. In total, 81 patients were randomly assigned to US commercial cetuximab plus chemotherapy (Arm A) and 73 patients were randomly assigned to BI-manufactured cetuximab plus chemotherapy (Arm B) in the two-arm, randomized, double-blind phase of the study (Fig. 1). Of these, 77 patients in Arm A and 71 patients in Arm B received at least one dose of any study drug and constituted the RT population. In Arm B, 9/71 patients (12.7\%) switched from BImanufactured cetuximab to US commercial cetuximab following the expiration of BI-manufactured cetuximab.

\section{Demographic and baseline clinical characteristics}

Baseline characteristics were mostly similar between Arm A and Arm B (Table 1). The majority of patients in the RT population were male (Arm A: 68/77 patients, $88.3 \%$; Arm B: 55/71 patients, $77.5 \%$ ). Median age was 57.8 and 61.3 years in Arm A and Arm B, respectively. The primary tumor site was the oral cavity/oropharynx in 48/77 patients (62.3\%) in Arm A and 45/71 patients (63.4\%) in Arm B, and the larynx/hypopharynx in $18 / 77$ patients $(23.4 \%)$ in Arm A and 13/71 patients (18.3\%) in Arm B.

\section{Exposure to cetuximab and chemotherapy}

Overall, the treatment durations of cetuximab and chemotherapy were mostly similar between Arm A and Arm B (Table 2). The relative dose intensity of cetuximab, cisplatin, and 5-FU was similar in the two arms; 


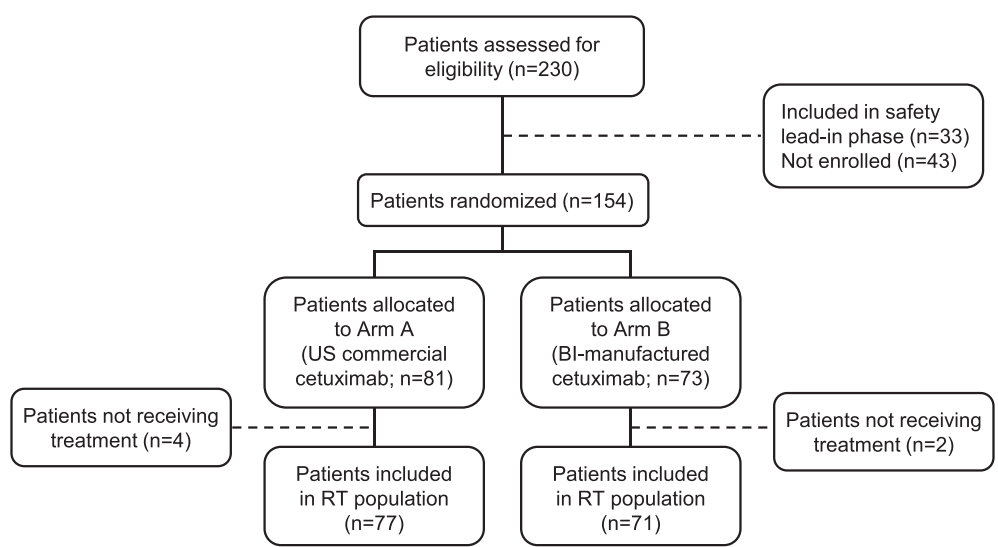

Fig. 1 Patient flow. Abbreviations: $B \mathrm{~B}=$ Boehringer Ingelheim; RT = randomized and treated; US = United States

the relative dose intensity of carboplatin was numerically higher in Arm A than Arm B (94.3\% vs 88.4\%).

\section{Safety}

There were no clinically meaningful differences in safety between Arm A and Arm B, as assessed by incidence of

Table 1 Patient demographics and baseline disease characteristics

\begin{tabular}{|c|c|c|}
\hline Characteristic & $\begin{array}{l}\text { US commercial } \\
\text { cetuximab } \\
\text { Arm A } \\
(\mathrm{N}=77)\end{array}$ & $\begin{array}{l}\text { Bl-manufactured } \\
\text { cetuximab } \\
\text { Arm B } \\
(\mathrm{N}=71)\end{array}$ \\
\hline \multicolumn{3}{|l|}{ Sex, n (\%) } \\
\hline Male & $68(88.3)$ & $55(77.5)$ \\
\hline \multicolumn{3}{|l|}{ Age, years } \\
\hline Median (range) & $57.8(26.9-77.2)$ & $61.3(29.2-81.9)$ \\
\hline \multicolumn{3}{|l|}{ KPS score, n (\%) } \\
\hline 70 & $9(11.7)$ & $7(9.9)$ \\
\hline 80 & $28(36.4)$ & $22(31.0)$ \\
\hline 90 & $32(41.6)$ & $31(43.7)$ \\
\hline 100 & $8(10.4)$ & $11(15.5)$ \\
\hline \multicolumn{3}{|l|}{ Stage of disease, n (\%) } \\
\hline Local & $8(10.4)$ & $12(16.9)$ \\
\hline Locoregional & $26(33.8)$ & $20(28.2)$ \\
\hline Metastatic & $43(55.8)$ & $38(53.5)$ \\
\hline \multicolumn{3}{|l|}{ Histologic type, n (\%) } \\
\hline Well differentiated & $3(3.9)$ & $6(8.5)$ \\
\hline Moderately differentiated & $35(45.5)$ & $29(40.8)$ \\
\hline Poorly differentiated & $25(32.5)$ & $17(23.9)$ \\
\hline Undifferentiated & $3(3.9)$ & $1(1.4)$ \\
\hline Unable to determine & $11(14.3)$ & $15(21.1)$ \\
\hline Missing/unknown & 0 & $2(2.8)$ \\
\hline
\end{tabular}

Abbreviations: BI Boehringer Ingelheim, KPS Karnofsky Performance Status, US United States (i) all-grade, all-cause TEAEs (the primary objective of the study), (ii) maximum CTCAE grade AEs regardless of causality, and (iii) maximum CTCAE grade AEs possibly related to study drug.

\section{All-grade, all-cause TEAEs}

The majority of patients in both arms experienced at least 1 TEAE, regardless of causality: 75/77 patients (97.4 \%) in Arm A and 68/71 patients (95.8 \%) in Arm B (Table 3). The TEAEs with the highest incidence in the two arms included nausea (Arm A: 38/77 patients, 49.4\%; Arm B: 34/71 patients, $47.9 \%$ ), fatigue (Arm A: 35/77 patients, $45.5 \%$; Arm B: 36/71 patients, $50.7 \%$ ), and hypomagnesemia (Arm A: 29/77 patients, $37.7 \%$; Arm B: $29 / 71$ patients, $40.8 \%$ ). There were no significant differences between Arm A and Arm B in the incidence of any individual TEAE, apart from the incidence of rash, which was significantly lower in Arm A than

Table 2 Summary of treatment exposure

\begin{tabular}{lll}
\hline Treatment exposure & $\begin{array}{l}\text { US commercial } \\
\text { cetuximab } \\
\text { Arm A } \\
(\mathrm{N}=77)\end{array}$ & $\begin{array}{l}\text { Bl-manufactured } \\
\text { cetuximab } \\
\text { Arm B } \\
(\mathrm{N}=71)\end{array}$ \\
\hline $\begin{array}{l}\text { Median duration of treatment } \\
\left(25^{\text {th }} \text { percentile, } 75^{\text {th }} \text { percentile), }\right. \\
\text { weeks }\end{array}$ & \\
Cetuximab & $12.4(6.0,18.0)$ & $13.6(6.0,20.0)$ \\
Cisplatin & $6.9(3.0,14.0)$ & $9.0(3.0,18.0)$ \\
Carboplatin & $12.6(6.1,18.0)$ & $15.0(7.0,20.0)$ \\
5-FU & $13.0(6.0,17.9)$ & $13.0(6.3,20.0)$ \\
Relative dose intensity, \% & & \\
Cetuximab & 87.5 & 87.1 \\
Cisplatin & 86.8 & 87.9 \\
Carboplatin & 94.3 & 88.4 \\
5-FU & 84.1 & 82.9 \\
\hline
\end{tabular}

Abbreviations: 5-FU 5-fluorouracil, BI Boehringer Ingelheim, US United States 
Table 3 All-cause treatment-emergent adverse events occurring in $\geq 10 \%$ of patients in either arm

\begin{tabular}{|c|c|c|}
\hline Adverse event ${ }^{a}$ & $\begin{array}{l}\text { US commercial } \\
\text { cetuximab } \\
\text { Arm A } \\
(\mathrm{N}=77) \\
\mathrm{n}(\%)\end{array}$ & $\begin{array}{l}\text { Bl-manufactured } \\
\text { cetuximab } \\
\text { Arm B } \\
(\mathrm{N}=71) \\
\mathrm{n}(\%)\end{array}$ \\
\hline Patients with $\geq 1$ TEAE & 75 (97.4) & 68 (95.8) \\
\hline Nausea & $38(49.4)$ & $34(47.9)$ \\
\hline Fatigue & $35(45.5)$ & $36(50.7)$ \\
\hline Hypomagnesemia & $29(37.7)$ & $29(40.8)$ \\
\hline Diarrhea & $25(32.5)$ & $22(31.0)$ \\
\hline Dermatitis acneiform & $25(32.5)$ & $19(26.8)$ \\
\hline Vomiting & $24(31.2)$ & $27(38.0)$ \\
\hline Anemia & $21(27.3)$ & $30(42.3)$ \\
\hline Hypokalemia & $21(27.3)$ & $24(33.8)$ \\
\hline Neutropenia & $20(26.0)$ & $24(33.8)$ \\
\hline Stomatitis & $20(26.0)$ & $21(29.6)$ \\
\hline Dry skin & $18(23.4)$ & $12(16.9)$ \\
\hline Constipation & $17(22.1)$ & $24(33.8)$ \\
\hline Mucosal inflammation & $16(20.8)$ & $16(22.5)$ \\
\hline Decreased appetite & $15(19.5)$ & $18(25.4)$ \\
\hline Dehydration & $13(16.9)$ & $17(23.9)$ \\
\hline Neutrophil count decreased & $11(14.3)$ & $14(19.7)$ \\
\hline Rash & $10(13.0)$ & $19(26.8)$ \\
\hline Weight decreased & $10(13.0)$ & $13(18.3)$ \\
\hline Platelet count decreased & $10(13.0)$ & $11(15.5)$ \\
\hline Insomnia & $9(11.7)$ & $11(15.5)$ \\
\hline Dyspnea & $8(10.4)$ & $12(16.9)$ \\
\hline Thrombocytopenia & $8(10.4)$ & $12(16.9)$ \\
\hline Dizziness & $8(10.4)$ & $11(15.5)$ \\
\hline Paronychia & $8(10.4)$ & $10(14.1)$ \\
\hline Hyponatremia & $7(9.1)$ & $14(19.7)$ \\
\hline Pyrexia & $7(9.1)$ & 8 (11.3) \\
\hline
\end{tabular}

${ }^{a}$ Apart from rash $(p=0.034,95 \% \mathrm{Cl}: 0.010,0.265)$, the difference between Arm $A$ and Arm B in the incidence of all-cause TEAEs occurring in $\geq 10 \%$ of patients in either arm was not statistically significant

Abbreviations: BI Boehringer Ingelheim, TEAE treatment-emergent adverse event, US United States

Arm B. The TEAE of rash was reported in 10/77 patients (13.0 \%) in Arm A and 19/71 patients (26.8\%) in Arm B ( $p=0.034,95$ \% CI: 0.010, 0.265).

\section{Analysis of adverse events by maximum CTCAE grade}

When AEs were analyzed by maximum CTCAE grade, the percentage of patients who experienced at least $1 \mathrm{AE}$ regardless of causality was similar in the two arms: 76/ 77 patients (98.7 \%) in Arm A and 68/71 patients (95.8 \%) in Arm B (absolute risk difference between the two arms: $0.029 ; p=0.281,95 \% \mathrm{CI}$ : $-0.024,0.082)$. The incidence of bone pain, febrile neutropenia, laryngeal hemorrhage, somnolence, and syncope was significantly lower in Arm A than Arm B (Table 4). The percentage of patients who experienced at least $1 \mathrm{AE}$ possibly related to study drug was also similar in the two arms: 69/77 patients (89.6\%) in Arm A and 64/71 patients (90.1\%) in Arm B (absolute risk difference between the two arms: $0.005 ; p=0.915,95 \%$ CI: $-0.092,0.103)$. The incidence of dysguesia and febrile neutropenia possibly related to study drug was significantly lower in Arm A than Arm B (Table 4).

\section{Adverse events of special interest for cetuximab treatment}

There were no significant differences between Arm A and Arm B in the incidence of acneiform rash, cardiac events, infusion reactions, or hypomagnesemia (Table 5). A little over half of the patients in each arm reported acneiform rash possibly related to study drug, mostly grade 1 or 2 in severity. Grade 3 skin reactions possibly related to study drug were dermatitis acneiform (Arm A: 4/77 patients, $5.2 \%$; Arm B: 4/71 patients, $5.6 \%$ ) and rash (Arm A: no patients; Arm B: 3/71 patients, $4.2 \%$ ). Two patients in Arm A died of cardiac arrest; one death from cardiac arrest was considered possibly related to study drug. No grade 3 or 4 cardiac events were reported in Arm A and no grade 4 or 5 cardiac events were reported in Arm B. Few patients reported grade 3 or 4 infusion reactions (Table 6). In Arm A, 2 patients (2.6\%) experienced grade 4 anaphylactic shock and 1 patient $(1.3 \%)$ experienced a grade 4 infusion-related reaction; these events were possibly related to study drug.

Table 4 Summary of adverse events that significantly differed in incidence between arms by maximum CTCAE grade

\begin{tabular}{|c|c|c|c|}
\hline Event & $\begin{array}{l}\text { US commercial } \\
\text { cetuximab } \\
\text { Arm A } \\
(\mathrm{N}=77) \\
\mathrm{n}(\%)\end{array}$ & $\begin{array}{l}\text { Bl-manufactured } \\
\text { cetuximab } \\
\text { Arm B } \\
(\mathrm{N}=71) \\
\mathrm{n}(\%)\end{array}$ & $\begin{array}{l}\text { Arm A vs Arm B } \\
p \text { value }(95 \% \mathrm{Cl})\end{array}$ \\
\hline \multicolumn{4}{|l|}{$\begin{array}{l}\text { Regardless of } \\
\text { causality }\end{array}$} \\
\hline Bone pain & $2(2.6)$ & $9(12.7)$ & $0.020(0.016,0.186)$ \\
\hline $\begin{array}{l}\text { Febrile } \\
\text { neutropenia }\end{array}$ & $1(1.3)$ & $11(15.5)$ & $0.002(0.054,0.230)$ \\
\hline $\begin{array}{l}\text { Laryngeal } \\
\text { hemorrhage }\end{array}$ & $0(0.0)$ & $6(8.5)$ & $0.010(0.020,0.149)$ \\
\hline Somnolence & $0(0.0)$ & $4(5.6)$ & $0.040(0.003,0.110)$ \\
\hline Syncope & $0(0.0)$ & $5(7.0)$ & $0.020(0.011,0.130)$ \\
\hline \multicolumn{4}{|c|}{$\begin{array}{l}\text { Possibly related to } \\
\text { study drug }\end{array}$} \\
\hline Dysgeusia & $1(1.3)$ & $6(8.5)$ & $0.044(0.002,0.141)$ \\
\hline $\begin{array}{l}\text { Febrile } \\
\text { neutropenia }\end{array}$ & $1(1.3)$ & $8(11.3)$ & $0.012(0.022,0.177)$ \\
\hline
\end{tabular}

Abbreviations: $B$ I Boehringer Ingelheim, $C$ confidence interval, CTCAE Common Terminology Criteria for Adverse Events, US United States 
Table 5 Treatment-emergent adverse events of special interest for cetuximab

\begin{tabular}{|c|c|c|c|c|}
\hline Adverse event & Relatedness of adverse event & $\begin{array}{l}\text { US commercial cetuximab } \\
\text { Arm A } \\
(\mathrm{N}=77) \\
\mathrm{n}(\%)\end{array}$ & $\begin{array}{l}\text { Bl-manufactured cetuximab } \\
\text { Arm B } \\
(\mathrm{N}=71) \\
\mathrm{n}(\%)\end{array}$ & $\begin{array}{l}\text { Arm A vs Arm B } \\
p \text { value }(95 \% \text { Cl) }\end{array}$ \\
\hline \multirow[t]{2}{*}{ Acneiform rash ${ }^{a}$} & Regardless of causality & $42(54.5)$ & $40(56.3)$ & $0.826(-0.142,0.178)$ \\
\hline & Possibly related to study drug & $41(53.2)$ & $40(56.3)$ & $0.706(-0.129,0.191)$ \\
\hline \multirow[t]{2}{*}{ Cardiac event ${ }^{\mathrm{b}}$} & Regardless of causality & $5(6.5)$ & $10(14.1)$ & $0.128(-0.022,0.174)$ \\
\hline & Possibly related to study drug & $2(2.6)$ & $6(8.5)$ & $0.120(-0.015,0.132)$ \\
\hline \multirow[t]{2}{*}{ Infusion reaction ${ }^{c}$} & Regardless of causality & $6(7.8)$ & $5(7.0)$ & $0.862(-0.077,0.092)$ \\
\hline & Possibly related to study drug & $5(6.5)$ & $4(5.6)$ & $0.826(-0.068,0.085)$ \\
\hline \multirow[t]{2}{*}{ Hypo-magnesemia } & Regardless of causality & $29(37.7)$ & $29(40.8)$ & $0.692(-0.126,0.189)$ \\
\hline & Possibly related to study drug & $26(33.8)$ & $23(32.4)$ & $0.859(-0.138,0.165)$ \\
\hline
\end{tabular}

${ }^{a}$ Composite term of 16 MedDRA Preferred Terms including dermatitis acneiform and rash

b Composite term of 39 MedDRA Preferred Terms including cardiac arrest and myocardial infarction

c Composite term of 9 MedDRA Preferred Terms including infusion-related reaction and anaphylactic shock

Abbreviations: BI Boehringer Ingelheim, Cl confidence interval, MedDRA Medical Dictionary for Regulatory Activities, US United States

No grade 4 infusion reaction events were reported in Arm B. Approximately one-third of patients in each arm reported hypomagnesemia possibly related to study drug, mostly grade 1 or 2 in severity. Grade 3 hypomagnesemia possibly related to study drug occurred in $3 / 77$ patients (3.9\%) in Arm A and 3/71 patients (4.2\%) in Arm B.

\section{Deaths}

In Arm A, there were 11 deaths during the study, of which 3 deaths were considered possibly related to study drug (cardiac arrest, hemorrhage intracranial, and septic shock). In Arm B, there were 7 deaths during the study,

Table 6 Grade 3/4 infusion reactions

\begin{tabular}{llll}
\hline Adverse event & $\begin{array}{l}\text { Relatedness of } \\
\text { adverse event }\end{array}$ & $\begin{array}{l}\text { US commercial } \\
\text { cetuximab } \\
\text { Arm A } \\
(\mathrm{N}=77) \\
\mathrm{n}(\%)\end{array}$ & $\begin{array}{l}\text { Bl-manufactured } \\
\text { cetuximab } \\
\text { Arm B } \\
(\mathrm{N}=71) \\
\mathrm{n}(\%)\end{array}$ \\
\hline $\begin{array}{llll}\text { Infusion-related } \\
\text { reaction }\end{array}$ & $\begin{array}{l}\text { Regardless of } \\
\text { causality } \\
\text { Possibly related } \\
\text { to study drug }\end{array}$ & $1(1.3)$ & $1(1.4)$ \\
Anaphylactic & $\begin{array}{l}\text { Regardless of } \\
\text { shock }\end{array}$ & $3(3.9)$ & $1(1.4)$ \\
causality & $\begin{array}{l}\text { Possibly related } \\
\text { to study drug }\end{array}$ & $3(3.9)$ & $0(0)$ \\
Hypersensitivity & $\begin{array}{l}\text { Regardless of } \\
\text { reaction }\end{array}$ & $0(0)$ & $1(1.4)$ \\
& $\begin{array}{l}\text { causality } \\
\text { tossibly related }\end{array}$ & $0(0)$ & $1(1.4)$ \\
Pyrexia & $\begin{array}{l}\text { Regardless of } \\
\text { causality }\end{array}$ & $0(0)$ & $0(0)$ \\
& $\begin{array}{l}\text { Possibly related } \\
\text { to study drug }\end{array}$ & $0(0)$ & $0(0)$ \\
\hline
\end{tabular}

Abbreviations: BI Boehringer Ingelheim, US United States of which 1 death was considered possibly related to study drug (septic shock).

\section{Efficacy}

There were no clinically meaningful differences in efficacy between Arm A and Arm B; median OS, median PFS, ORR, and DCR were similar in the two arms (Table 7).

\section{Discussion}

Overall, this study showed no clinically meaningful differences in safety between patients receiving US commercial cetuximab (Arm A) and those receiving BImanufactured cetuximab (Arm B), using the same therapeutic regimen as the EXTREME study [3]. The combination of US commercial cetuximab with cisplatin or carboplatin plus 5-FU did not result in any unexpected safety signals in the treatment of patients with locoregionally recurrent and/or metastatic SCCHN. The AEs reported in this study are consistent with the known safety profile of cetuximab, cisplatin/carboplatin, or 5-FU, or with the underlying disease.

The safety profile of US commercial cetuximab (Arm A) was similar to that of BI-manufactured cetuximab (Arm B). All-grade, all-cause TEAEs with the highest incidences included nausea, fatigue, and hypomagnesemia in both arms. There were no AEs for which the incidence was significantly higher in patients receiving US commercial cetuximab than those receiving BI-manufactured cetuximab. Conversely, the incidence of rash, bone pain, febrile neutropenia, laryngeal hemorrhage, somnolence, syncope (regardless of causality), dysguesia, and febrile neutropenia (possibly related to study drug) was significantly lower in patients receiving US commercial cetuximab than those receiving BI-manufactured cetuximab. These betweengroup differences were not considered clinically meaningful. The safety profile of US commercial cetuximab in 
Table 7 Summary of efficacy outcomes

\begin{tabular}{llll}
\hline Outcome & US commercial cetuximab & Bl-manufactured cetuximab & $\begin{array}{l}\text { Unadjusted HR } \\
\text { Arm A vs Arm B } \\
(95 \% \mathrm{Cl} ; p \text { value })\end{array}$ \\
\hline OS (months), median $(95 \% \mathrm{Cl})$ & $\begin{array}{l}\text { Arm B } \\
(\mathrm{N}=77)\end{array}$ & $9.46(6.87,11.43)$ & $0.92(0.619,1.368 ; p=0.681)$ \\
PFS (months), median $(95 \% \mathrm{Cl})$ & $4.23(7.10,11.80)$ & $5.65(4.04,6.47)$ & $1.04(0.717,1.497 ; p=0.850)$ \\
Overall response rate, $\%(95 \% \mathrm{Cl})$ & $29.9(19.6,40.1)$ & $36.6(25.4,47.8)$ & $\mathrm{NA}$ \\
Disease control rate, $\%(95 \% \mathrm{Cl})$ & $58.4(47.4,69.4)$ & $62.0(50.7,73.3)$ & $\mathrm{NA}$ \\
Complete response, $\mathrm{n}(\%)$ & $3(3.9)$ & $3(4.2)$ & $\mathrm{NA}$ \\
Partial response, $\mathrm{n}(\%)$ & $20(26.0)$ & $23(32.4)$ & $\mathrm{NA}$ \\
Stable disease, $\mathrm{n}(\%)$ & $22(28.6 \%)$ & $18(25.4 \%)$ & $\mathrm{NA}$ \\
\hline
\end{tabular}

Abbreviations: $B$ I Boehringer Ingelheim, CI confidence interval, HR hazard ratio, NA not applicable, OS overall survival, PFS progression-free survival, US United States

combination with chemotherapy in the current study is also consistent with that previously observed for BImanufactured cetuximab in combination with the same chemotherapy regimen in the EXTREME study [3]. Commonly reported AEs ( $>10 \%)$ in both studies included neutropenia, thrombocytopenia, and anemia, which are typically associated with cisplatin/carboplatin and 5-FU treatment [8-10]. In the current study, the incidence of febrile neutropenia (by maximum CTCAE grade and by grade $3 / 4$ ) was $1.3 \%$ in patients receiving US commercial cetuximab and $15.5 \%$ in patients receiving BImanufactured cetuximab. In the EXTREME study, the incidence of grade $3 / 4$ febrile neutropenia was $5 \%$ in both the cetuximab plus chemotherapy and chemotherapyalone groups [3].

There were no significant differences in the incidence of AEs of special interest for cetuximab (acneiform rash, cardiac events, infusion reactions, and hypomagnesemia) [5] between patients receiving US commercial cetuximab and those receiving BI-manufactured cetuximab. As reported in other studies of cetuximab $[3,4,11]$, the majority of skin reactions reported in both arms in the current study were grade 1 or 2 in severity. Overall, the frequency and nature of cardiac events, infusion reactions, and hypomagnesemia observed in patients receiving US commercial cetuximab in the current study are consistent with the known safety profile of cetuximab [5]. In the EXTREME study, the incidence of cardiac events was approximately $9 \%$ in both the cetuximab plus chemotherapy group and the chemotherapy-alone group; the incidence of death attributed to cardiovascular death or sudden death was $3 \%$ and $2 \%$ in the cetuximab plus chemotherapy group and chemotherapy-alone group, respectively [5]. In the same study, the incidence of hypomagnesemia was $14 \%$ and $6 \%$ in the subset of patients receiving cetuximab plus cisplatin and 5-FU and the subset of patients receiving cisplatin and 5-FU alone, respectively; the incidence of grade $3 / 4$ hypomagnesemia was $7 \%$ and $2 \%$, respectively [5].
Compliance and study treatment exposure to cetuximab, as assessed by median duration of treatment and cumulative dose of cetuximab, were similar in the two arms. The relative dose intensity was more than $85 \%$ for both US commercial cetuximab and BI-manufactured cetuximab during the combination chemotherapy period. The relative dose intensities of cisplatin, carboplatin, and 5-FU were also high in both treatment arms, ranging from around $80 \%$ to $95 \%$, suggesting that the various combinations of agents used in this study were well tolerated. These results are consistent with the observation in the EXTREME study that the addition of cetuximab to cisplatin/carboplatin plus 5-FU did not affect the tolerability of the chemotherapy regimen [3]. In the EXTREME study, the median (interquartile range) duration of cisplatin and carboplatin treatment in the cetuximab arm was 15 weeks (6 to 19 ) and 18 weeks (10 to 19), respectively [3]. The relative dose intensity of cisplatin was $\geq 80 \%$ in $89 \%$ of patients and the relative dose intensity of carboplatin was $\geq 80 \%$ in $93 \%$ of patients in the cetuximab arm in the EXTREME study [3].

There were no clinically meaningful differences in OS, PFS, ORR, or DCR between patients receiving US commercial cetuximab and those receiving BI-manufactured cetuximab. While it should be noted that the current study was not designed or powered to assess efficacy, the efficacy results of this study are consistent with those reported in the EXTREME study. Median OS with US commercial cetuximab and BI-manufactured cetuximab was 9.23 months and 9.46 months, respectively, in the current study and 10.1 months with BI-manufactured cetuximab in the EXTREME study [3]. Median PFS with US commercial cetuximab and BI-manufactured cetuximab was 4.70 months and 5.65 months, respectively, in the current study and 5.6 months with BI-manufactured cetuximab in the EXTREME study [3]. The ORR with US commercial cetuximab and BI-manufactured cetuximab was $29.9 \%$ and $36.6 \%$, respectively, in the current 
study and $36 \%$ with BI-manufactured cetuximab in the EXTREME study.

A strength of the current study was analyzing the safety of two formulations of a drug in a randomized controlled trial. A limitation of the study was that the sample size was based on practical and clinical considerations. This was done to ensure that assessment of the safety profile could be appropriately compared between the two treatment arms, rather than on any statistical assumptions or hypotheses. However, the planned sample size was not met because the supply of BI-manufactured cetuximab under evaluation expired during the study. In addition, not all patients in Arm B received BI-manufactured cetuximab for the duration of the study: 9/71 patients in Arm B were switched to US commercial cetuximab when the supply of BI-manufactured cetuximab expired. The potential bias that this may have introduced was addressed by choosing the earliest date that a patient on Arm B was switched from BI-manufactured cetuximab to US commercial cetuximab as the cut-off date for the primary safety analysis. However, this early cut-off date also limited the duration of the safety period for this analysis.

\section{Conclusions}

The safety profile of US commercial cetuximab in patients with locoregionally recurrent and/or metastatic SCCHN is consistent with the safety profile of BI-manufactured cetuximab in the current study and that reported in the previously published EXTREME study [3]. The combination of US commercial cetuximab with cisplatin or carboplatin plus 5-FU did not result in any unexpected safety signals; the AEs reported in this study are consistent with the known safety profile of cetuximab, cisplatin/carboplatin, or 5-FU, or with the underlying disease. In addition, there were no clinically meaningful differences in OS, PFS, or ORR between patients receiving US commercial cetuximab and those receiving BI-manufactured cetuximab. These results indicate that, despite potentially higher systemic exposures with US commercial cetuximab relative to BImanufactured cetuximab, the safety profile of US commercial cetuximab is consistent with the safety profile of cetuximab in the current US prescribing information [5].

\footnotetext{
Abbreviations

AE: adverse event; AUC: area under the concentration curve; BI: Boehringer Ingelheim; Cl: confidence interval; CR: complete response; CTCAE: Common Terminology Criteria for Adverse Events; DCR: disease control rate; EGFR: epidermal growth factor receptor; EU: European Union; EXTREME: Erbitux in First-Line Treatment of Recurrent or Metastatic Head and Neck Cancer; FDA: Food and Drug Administration; 5-FU: 5-fluorouracil; IVRS: interactive voice response system; KPS: Karnofsky Performance Status; MedDRA: Medical Dictionary for Regulatory Activities; ORR: overall response rate; OS: overall survival; PFS: progression-free survival; PR: partial response; RECIST: Response Evaluation Criteria in Solid Tumors; RT: randomized and treated; SCCHN: squamous cell carcinoma of the head and neck; TEAE: treatment-emergent adverse event; US: United States.
}

\section{Competing interests}

KB, SH, CKO, S-CC, and SC are employees of Eli Lilly and Company, and $\mathrm{HL}$ was an employee of Eli Lilly and Company at the time the study was conducted. CKO owns stock in Eli Lilly and Company. DS, KM, and DA have received research funding for their institution from Eli Lilly and Company. DS has participated in advisory boards for Pfizer, Roche, Novartis, Celgene, and Merck \& Co., and has received research funding for his institution from more than 20 companies. DA has received research funding from Celgene, Pfizer, GlaxoSmithKline, Novartis, Galera Therapeutics, Soligenix, Inc., Mirati Therapeutics, Merck \& Co., AstraZeneca, and VentiRX Pharmaceuticals. $J \mathrm{~A}, \mathrm{EC}$, and SE have no conflicts of interest to declare.

\section{Authors' contributions}

All authors participated in the interpretation of study results, and in the drafting, critical revision, and approval of the final version of the manuscript. All authors have read and approved the manuscript. HL, KB, CKO, S-CC, SC, and DA were involved in the study design. DS, JLA, EC, KM, SE, and DA were investigators in the study. SH conducted the statistical analysis. KM and DA were involved in the data collection for the study.

\section{Acknowledgements}

This study was sponsored by Eli Lilly and Company. US commercial cetuximab is manufactured by ImClone LLC, a wholly-owned subsidiary of Eli Lilly and Company. Medical writing assistance was provided by Justine Southby, PhD, CMPP, and Serina Stretton, PhD, CMPP, of ProScribe - Envision Pharma Group, and was funded by Eli Lilly. ProScribe's services complied with international guidelines for Good Publication Practice (GPP2).

Eli Lilly was involved in the study design, data collection, data analysis, and preparation of the manuscript.

\section{Author details}

${ }^{1}$ Centre Hospitalier de I'Université de Montréal, Montréal, Québec, Canada. ${ }^{2}$ Instituto Nacional de Cancerologia, Mexico City, Mexico. ${ }^{3}$ Princess Margaret Hospital, Toronto, Ontario, Canada. ${ }^{4}$ Mount Sinai School of Medicine Tisch Cancer Institute, New York, NY, USA. ${ }^{5}$ London Regional Cancer Center, London, Ontario, Canada. ${ }^{6}$ Eli Lilly and Company, Indianapolis, IN, USA. ${ }^{7}$ Washington University School of Medicine, 660 S. Euclid, Box 8056, St. Louis, MO 63110, USA

Received: 16 April 2015 Accepted: 10 January 2016

Published online: 14 January 2016

\section{References}

1. Vermorken JB, Specenier P. Optimal treatment for recurrent/metastatic head and neck cancer. Ann Oncol. 2010;21 Suppl 7:vii252-61.

2. National Comprehensive Cancer Network. NCCN clinical practice guidelines in oncology: head and neck cancer. Version 2.2014. http://www.nccn.org/ professionals/physician_gls/pdf/head-and-neck.pdf. Accessed 24 Nov 2014.

3. Vermorken JB, Mesia R, Rivera F, Remenar E, Kawecki A, Rottey S, et al. Platinum-based chemotherapy plus cetuximab in head and neck cancer. N Engl J Med. 2008;359:1116-27.

4. Burtness B, Goldwasser MA, Flood W, Mattar B, Forastiere AA. Phase III randomized trial of cisplatin plus placebo compared with cisplatin plus cetuximab in metastatic/recurrent head and neck cancer: an Eastern Cooperative Oncology Group study. J Clin Oncol. 2005;23:8646-54.

5. Erbitux Prescribing Information. Revised August 2013. Branchburg, NJ: ImClone LLC, a wholly-owned subsidiary of Eli Lilly and Company. http://packageinserts.bms.com/pi/pi_erbitux.pdf. Accessed 11 Nov 2014.

6. Erbitux Summary of Product Characteristics. Revised August 2014. Darmstadt, Germany: Merck KGaA. http://www.ema.europa.eu/docs/en_ GB/document_library/EPAR_-_Product_Information/human/000558/ WC500029119.pdf. Accessed 26 Nov 2014.

7. A Study in Head and Neck Cancer. ClinicalTrials.gov study record. http://clinicaltrials.gov/show/NCT01081041. Accessed 27 Nov 2014.

8. Platinol [product information]. Bristol-Myers Squibb Company 2010. http://www.accessdata.fda.gov/drugsatfda_docs/label/2011/018057s080lbl.pdf. Accessed 11 Nov 2014.

9. Carboplatin FDA Professional Drug Information. http://www.drugs.com/pro/ carboplatin.html. Accessed 14 Nov 2016. 
10. Adrucil (Fluorouracil) Injection. TEVA Parenteral Medicines, Inc. http://dailymed. nlm.nih.gov/dailymed/druglnfo.cfm?setid=e0794add-67a7-4308-93e9f889472716cc. Accessed 11 Nov 2014.

11. Bourhis J, Rivera F, Mesia R, Awada A, Geoffrois L, Borel C, et al. Phase I/II study of cetuximab in combination with cisplatin or carboplatin and fluorouracil in patients with recurrent or metastatic squamous cell carcinoma of the head and neck. J Clin Oncol. 2006;24:2866-72.

Submit your next manuscript to BioMed Central and we will help you at every step:

- We accept pre-submission inquiries

- Our selector tool helps you to find the most relevant journal

- We provide round the clock customer support

- Convenient online submission

- Thorough peer review

- Inclusion in PubMed and all major indexing services

- Maximum visibility for your research

Submit your manuscript at www.biomedcentral.com/submit
Biomed Central 